\title{
CORRELATION DURING THE FRACTURE PROCESS ANALYSED WITH THE HELP OF RIPLEY'S FUNCTIONS
}

\author{
DAVID GRÉGOIRE* , VINCENT LEFORT AND GILLES PIJAUDIER-CABOT \\ Université Pau \& Pays Adour \\ LFC-R, UMR5150 \\ 64600 Anglet, France \\ *corresponding author \\ e-mail: david.gregoire@univ-pau.fr
}

Key words: Fracture, Quasi-brittle materials, Fracture Process Zone, Boundary effect, Mesoscale, Mesoscopic model, Experimental, Acoustic emission, Ripley's functions

\begin{abstract}
The degradation of quasi-brittle materials encompasses micro-cracks propagation, interaction and coalescence in order to form a macro-crack. These phenomena are located within the Fracture Process Zone (FPZ). This paper aims at providing a further insight in the description of the FPZ evolution with the help of statistical analysis of damage. The statistical analysis relies on the implementation of Ripley's functions, which have been developed in order to exhibit patterns in image analyses. It is shown how a correlation length may be extracted from the Ripley's function analysis. Comparisons between experimental and numerical evolutions of extracted correlation lengths are performed.
\end{abstract}

\section{INTRODUCTION}

Fracture of quasi-brittle materials such as concrete or rocks is characterized by a macro crack surrounded by a damage zone. At the tip of the macro crack and ahead lies the so-called Fracture Process Zone (FPZ) which is a region of the material undergoing distributed damage. The size of the FPZ in these heterogeneous materials is large enough to influence the mechanical behaviour of the structure significantly. It does not depend on the structural size, but it is rather controlled by the local heterogeneities in the material as well as by the geometry of the specimen and the stress conditions. Therefore, size effect, understood here as the dependence of the dimensionless nominal strength of a structure on its size, is observed (e.g. when geometrically similar structures are compared, see for example [13]).
Experimentally, this damage zone may be characterized with the help of several direct and indirect techniques. The localization of acoustic events that can be detected during crack propagation is one well established technique from which the FPZ can be visualized and characterized (e.g. [8], [19], [21], [14]). The acoustic events generated during micro-cracking are recorded and post-processed in order to localize them with the help of time-of-flight algorithms. Hence, this technique provides information on the entire crack propagation process composed of distributed micro cracking and further coalescence into a macro crack. Haidar and co-workers [15] used a model mortar material to observe the correlation among the width of the FPZ measured by acoustic emissions analysis, the parameters entering in the description of size effect, and the so-called internal 
length used in classical non-local constitutive relations.

As far as modeling is concerned, macroscale approaches (e.g. continuum-based models) and mesoscale models (e.g. discrete or lattice-based models) are available. The first one involves a characteristic length, which controls the size of the FPZ and is more suited for classical structural analyses. In recent continuum-based macroscale models (see e.g. [6, 12, 17] among others), however, it has been pointed out that this internal length is not constant during the fracture process and also that it is influenced by boundaries, which could be expected since experimental works on fracture in concrete underline the influence of boundaries on the fracture energy [4]. The second approach relies on a mesoscale description of the material and on an explicit description of the heterogeneities in the material. Therefore, this approach is better suited in order to achieve an in-depth comprehension of the degradation processes involved during fracture. As opposed to the continuum macroscale approach, mesoscale models do not introduce a characteristic length to drive the failure process. The failure process is driven by the larger heterogeneities, which are explicitly represented in the meso-model. At the scale of a lattice element or a discrete element, softening is introduced as a local property. Note that continuum-based models may also be used at the mesoscale [18] by explicitly describing the heterogeneities and then may be also suited to achieve an in-depth comprehension of the degradation processes involved during fracture.

Grassl and co-workers [9] demonstrated that lattice-based mesoscale modeling was very efficient at describing not only size effect on the peak load, but also the entire load deflection response of bending beams. Four geometrically similar sizes and three different notch lengths were considered. The experimental data obtained by Grégoire et al. [13] could be quite accurately described, once the model parameters at the mesoscale level had been calibrated for one notch length. In addition, the authors used this model for studying the incremental distri- bution of the dissipated energy densities, and they were able to track the evolution of the fracture process zone in the structure, depending on the size of the beams and on the boundary conditions.

In addition, Grégoire and co-workers [14] demonstrated that this lattice-based mesoscale approach is also capable to capture the local failure process realistically. Three point bending experiments coupled with acoustic emission analyses provided global responses of the same bending beams and local data in the form of the distribution of the acoustic events and its evolution in the course of fracture. The experimental data obtained by Grégoire et al. [14], in term of energy dissipation maps and histograms of the distances between damage events, could be quite accurately described with the same set of model parameters. Particularly, the agreement between the distributions of the relative distances between damage events show that the mesoscale model depicts the fracture process zone and its evolution during failure in a very consistent way compared to acoustic emission data. Unfortunately, and contrary to the case of direct tension, these histograms cannot be interpreted easily because the effect of the strain gradient in bending beams cannot be easily separated from the interaction between damage events that may develop in the course of fracture.

The purpose of this paper is to provide a further insight in the description of failure with the help of statistical analyses of damage. The statistical analysis relies on the implementation of Ripley's functions [22], which have been developed in order to exhibit patterns in image analyses.

This paper is organized as follows: section 2 shows how Ripley's function may be used in the context of damage mechanics to extract a correlation length between damage events. Section 3 recalls briefly the lattice model used in this paper. Section 4 presents the comparison between the evolution of extracted correlation length during mesoscale numerical simulations and experimental three point bending tests 
where damage events are localized by acoustic emission techniques. Finally, numerical investigations of correlation length evolutions upon failure are presented in section 5 for both direct tension and three point bending specimens. Results show that the computed correlation length is not constant during failure and significant differences may be observed depending on the type of loading applied to the same specimen.

\section{RIPLEY'S FUNCTIONS APPLIED TO DAMAGE MECHANICS}

\subsection{Ripley's $K$ function description}

Ripley's $K$ function proposed in Ref. [22] is a tool for analyzing completely mapped spatial point process data, i.e. data on the locations of events [3]. Particularly, it is used to characterize the randomness in the spatial spreading of point distributions. It is of high interest in spatial ecology and has been used to characterize the development and spreading of different patterns, such as cell migration [22], tree [5] and plant [23] dissemination or disease spreading [2]. Recently, Tordesillas et al. [24] extended this pattern characterization method to non-biological system to analyze diffuse granular failure. In this paper, we will use the Ripley's $K$ function to characterize the interactions and the correlations induced by damage localization in quasibrittle fracture. The Ripley's $K$ function may be adapted to study one, two or three-dimensional spatial data, but most of the developments have been performed in $2 \mathrm{D}$, which will also be the case hereafter. For a spatial point distribution, the Ripley's $K$ function may be defined as the ratio between the density of events and the mean number of events within a distance $r$ of any chosen event in the distribution:

$$
\begin{gathered}
K(r)=\frac{1}{N \rho} \sum_{i \in \mathcal{P}} \sum_{j \in \mathcal{P}} e_{i j} H(i, j, r) \\
\text { and } H(i, j, r)=\left\{\begin{array}{l}
1 \text { if } D(i, j) \leq r \\
0 \text { if } D(i, j)>r
\end{array} .\right.
\end{gathered}
$$

In Eq. 1. $N$ is the total number of points, $\rho$ is the point density, $\mathcal{P}$ is the point distribution and $D(i, j)$ is the euclidean distance between two points $i$ and $j . e_{i j}$ is an edge effect correction factor, which is introduced to take into account that, for points located near the boundary of the study area, the real number of neighbors can be underestimated because some of them may be located outside of the study area or outside the specimen [7]. In 2D, the general expression of this edge effect correction factor is given by:

$$
e_{i j}=\frac{\pi D(i, j)}{\Pi_{\mathrm{int}}(i, j)} \geq 1 .
$$

In Eq. 2, $D(i, j)$ is the euclidean distance between two points $i$ and $j$ and $\Pi_{\text {int }}(i, j)$ is the inner perimeter corresponding to the part of the perimeter of the circle, centered at $i$ with a radius of $D(i, j)$, which is included inside the study area. This edge effect correction factor depends on the shape of the study area. Typical edge effect correction factor expressions for a rectangular study area may be found in [7].

\subsection{Randomness characterization}

The Ripley's $K^{\text {ran }}$ function of a perfect randomly distributed set of points is simply given by:

$$
K^{\mathrm{ran}}(r)=\pi r^{2} .
$$

In order to characterize the randomness of a distribution, the Ripley's $\mathrm{K}$ function is usually compared to this reference function $K^{\text {ran }}$ by defining the residual function $L$ as:

$$
L(r)=\sqrt{\frac{K(r)}{\pi}}-\sqrt{\frac{K^{\operatorname{ran}(r)}}{\pi}}=\sqrt{\frac{K(r)}{\pi}}-r .
$$

Within this definition, and for a randomly distributed set of points, the residual function stays equal to zero. Thus, plotting the residual $L$ for an arbitrary point distribution may characterize the distance of this distribution to a perfect random one and then characterize the randomness of the distribution (see [20] for illustrations). Applying this concept to a set of damage points may lead to characterize how these damage points localize upon failure and therefore characterize the correlations between these damage points, which are related to the internal length in a nonlocal continuum setting. 


\subsection{Correlation length extraction}

Figure 1 presents a distribution of 20 points located in a unique disk of radius $R_{0}=10 \mathrm{~mm}$ and the corresponding residual function $L$. The position of the maximum of the residual function $r_{\max } \approx 15.9 \mathrm{~mm}$ does not correspond directly to the disk radius since it depends also on the height and the length of the analyze area. However, the analytical expression of the residual function $L^{\text {disk }}$ of a distribution of events located in a disk of radius $R$ may be approximated by equation 5. For a given residual function $L$ of a distribution located in a unique disk, equation 6 provides the expression of the optimum radius $R^{*}$, which best fits the residual analytical function $L^{\text {disk }}$. This has been done for the distribution presented in Figure 1. One obtains $R^{*} \approx 9.57 \mathrm{~mm}$, which corresponds to an estimate of the disk radius with an error of less than $4.5 \%$ for this very coarse distribution.

$$
\begin{aligned}
L^{\text {disk }}(r, R) & = \begin{cases}\sqrt{\frac{S}{\pi R^{2}}}\left(r-\frac{r^{2}}{4 R}\right)-r & \text { for } \mathrm{r} \leq 2 R \\
\sqrt{\frac{S}{\pi}}-r & \text { for } \mathrm{r}>2 R\end{cases} \\
R^{*} & =\frac{1-\sqrt{1-2 r_{\max } \sqrt{\frac{\pi}{S}}}}{2 \sqrt{\frac{\pi}{S}}}
\end{aligned}
$$

where $S$ is the surface of the analysis box $\left(S=0.1 \times 0.1 \mathrm{~m}^{2}\right.$ in Figure 1$)$ and $r_{\max }$ is the position of the maximum of the residual function.

For a given distribution of damage events with no particular shape, we define the maximum $r_{\max }$ of the residual function $L(r)$ as the correlation length of the distribution. Thereby, this correlation length may be extracted directly from the evolution of the residual function.

Note that according to this definition, the extracted correlation length depends on the size of the analysis area. This dependance is attenuated for large analysis area $\left(R^{*} \rightarrow \frac{r_{\max }}{2}\right.$ when $S \rightarrow$ $+\infty$ in Eq. 6). Thereafter, all Ripley's functions are estimated for a sufficiently large analysis area of a given constant size $400 \times 400 \mathrm{~mm}$ and no edge effect correction is required. (a)

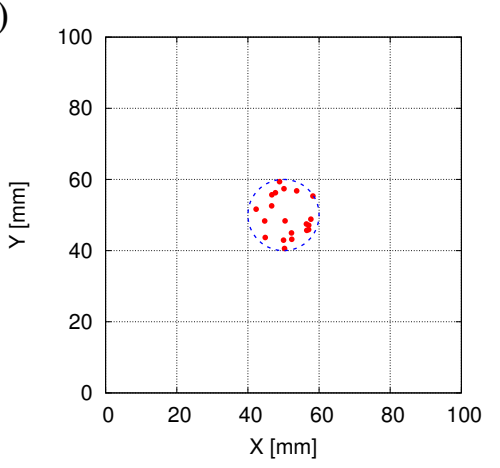

(b)

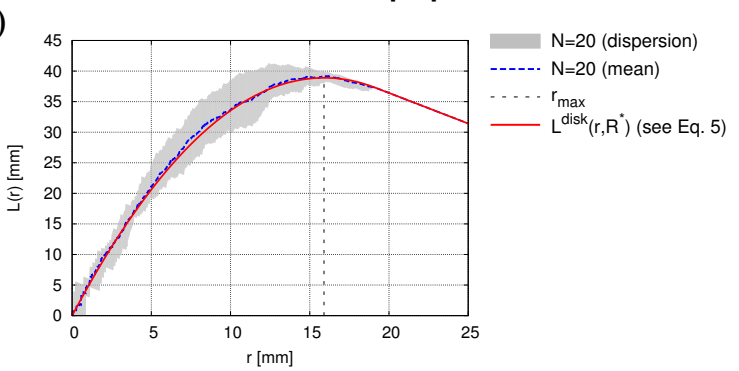

Figure 1: Distribution of events located in a disk of radius $R_{0}=10 \mathrm{~mm}$ (a) and corresponding residual function for 5 different analyses (b). Reproduced from [20].

\section{LATTICE MODEL DESCRIPTION}

A 2D plane-stress lattice model is used to characterize the correlations involved during failure in quasi-brittle materials. Practically, the lattice model is used to monitor an evolving population of damage events (a damage event corresponds to a lattice element undergoing damage during a load step), which is analyzed at each load step with the correlation length extraction method presented in part 2.3 and based on Ripley function applied to damage mechanics. This lattice model is based on the numerical framework proposed by Grassl and Jirasek [11]. It has been shown in previous study that this mesoscale approach is capable not only to provide consistent global responses (e.g. Force v.s. CMOD responses) [9, 14] but also to capture the local failure process realistically [14].

The lattice is made of beam elements and idealizes the meso-structure of concrete as a set of three different components: aggregates, $m a-$ trix and the interface between them. The following assumptions are used: 
(i) Aggregates are described as circular inclusions. Aggregates with a diameter $\phi^{d}$ greater than a fixed diameter value $\phi_{\min }^{d}$ are described explicitly. Their size distribution follows the grading of the concrete mixture and their spatial location is given by a random distribution defined by the cumulative distribution function proposed in Ref. [11]. Aggregates overlapping is avoided.

(ii) Fine aggregates are not described. They are included in the matrix which is an equivalent homogeneous material (made of cement paste and fine aggregates). Disorder due to the heterogeneity of the matrix which contains small aggregates is still kept, however, in the form of a correlated random distribution of mechanical properties. The correlation length is independent from the fineness of the lattice and therefore provides lattice element size independent results [10].

(iii) The large aggregate are elastic. The matrix material follows an isotropic - scalar damage model.

(iv) Each aggregate is surrounded by an interface of thickness equal to one lattice element length which is endowed with a special constitutive relation. This interface is meant to represent the Interfacial Transition Zone (ITZ) in concrete. Its constitutive model is similar to that of the matrix, with different constants since the ITZ is usually weaker than the matrix.

Model constitutive equations are not recalled here. The reader may refer to reference [9, 11, 14,20] for further details.

\section{MODEL VALIDATION BY EXPERIMEN- TAL COMPARISONS OF CORRELATION LENGTH EVOLUTIONS}

Three-point bending tests were performed on geometrically similar notched and unnotched specimens made of the same concrete material. The experimental results presented hereafter are obtained from a campaign already presented by Grégoire et al. [14]. This campaign is similar to the one previously presented by Grégoire et al. [13] and includes the localisation of acoustic events during fracture additionally. The experimental procedure is briefly presented in this section. The reader may refer to references [13, 14] for further details.

\subsection{Experimental procedure: material, specimen and test rig descriptions}

The concrete formulation used here is based on a ready-mix concrete mixture obtained from Unibeton . The concrete mixture formulation and detailed gradings of the sand, the aggregates and the mix are given in [13]. After demolding, the specimens were stored under water at $20^{\circ} \mathrm{C}$. The characterization of their mechanical properties was made by compression and splitting (Brazilian) tests according to $\mathrm{Eu}-$ ropean standards (EN 12390-1-3-6). Mechanical properties and mechanical responses of the material are presented in [13, 14, 20]. The testing rig used for the bending tests was a threepoint bending setup on a servo-hydraulic testing machine (HB250, Zwick/Roell) (see figure 2.a and figure 2 b).

Figure 2.c presents a sketch of the specimen geometry and the different measurable quantities. Three HN200 half-notched specimens $\left(D=200 \mathrm{~mm} ; a_{0}=0.5 D\right)$, three FN200 fifth-notched ( $\left.D=200 \mathrm{~mm} ; a_{0}=0.2 D\right)$, two UN200 unnotched ( $\left.D=200 \mathrm{~mm} ; a_{0}=0 \mathrm{~mm}\right)$ and three UN100 unnotched $(D=100 \mathrm{~mm}$; $\left.a_{0}=0 \mathrm{~mm}\right)$ have been tested. The thickness was kept constant $(50 \mathrm{~mm})$. All tests were CMOD controlled at an imposed velocity in order to avoid post-peak unstable crack propagation. Specimen dimensions and experimental conditions are detailed in [20].

\subsection{Acoustic Emission measurements}

During the tests, acoustic events were recorded and localised. The AE system used in this study comprised an eigth-channel MIS- 

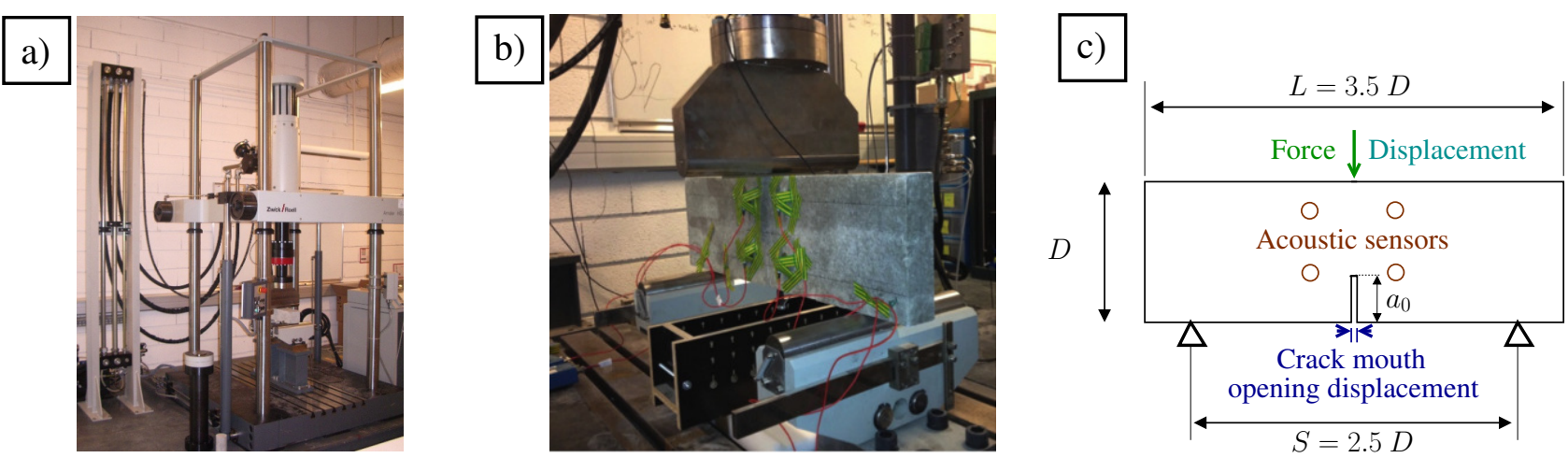

Figure 2: Photography of the servo-hydraulic testing machine (a), zoom on the test rig (b) and sketch of the specimen geometry and measurable quantities (c). Reproduced from [14].

TRAS system, a general purpose interface bus (PCI-DISP4) and a PC for data storage analysis. Four acoustic transducers (resonant frequency of $150 \mathrm{kHz}$ ) were placed around the expected location of the crack, on one side of specimen. The AE event localisation program relies on time of flight analysis and triangulation. The criterion used is that waves generated must reach at least 3 sensors. Then, the source location is determined by a $2 \mathrm{D}$ triangulation algorithm which relies of AEs arrival time and wave velocity. The details about $\mathrm{AE}$ setting parameters are given in [1]. Transducers were installed so that a minimum distance $d_{\min }=1.25 \mathrm{~cm}$ to the location where the crack could appear was respected in order to minimize errors which may occur when events are located near one sensor. Details on the transducers arrangement are given in [14].

The detected signals were then amplified with a $40 \mathrm{~dB}$ gain differential amplifier in a frequency band from 20 to $120 \mathrm{kHz}$. In order to limit the background noise, the signal detection threshold was set at a value of $35 \mathrm{~dB}$. The coupling between the transducer and the specimen is important in order to achieve a good accuracy of the localization of events. A thin layer of silicone grease was used to guarantee the correct transmission of acoustic signals between the beam and the transducer. The validation of both this coupling and the accuracy of the acoustic events localisation followed the European standard NF EN 1330. It consists in verifying if the position of an on-surface signal generated by the break of a short piece of pencil lead is correctly determined by the triangulation software. Thus, events were generated at several locations on the surface of each specimen and the results from the localisation software were compared with the true location of each event. A correct coupling is achieved when the accuracy of localization of these events is of the order of $4 \mathrm{~mm}$. Maps of the distribution of acoustic events for the different beam geometries are presented in [14].

\subsection{Numerical simulations: geometry, model parameter and global mechan- ical responses}

Figures 3,a and 3,b present a schematic drawing of the notched and unnotched beams considered in the present study. The geometry and applied loads correspond to the experiments reported in Refs. [13, 14] and modeled numerically in Refs. [9, 14]. Four different sizes of geometrically similar specimens were considered, along with three notch lengths: $a_{0}=0$ (UnNotched, so-called UN), $a_{0}=0.2 D$ (FifthNotched, so-called FN) and $a_{0}=0.5 D$ (HalfNotched, so-called HN). For a detailed presentation of the experiments, see [13, 14].

The analyses were controlled by the crack mouth opening displacement (CMOD), which is the relative horizontal displacement of the points $\mathrm{A}$ and $\mathrm{B}$ shown in Figures 3 a and 3 . b. For the notched specimens, the points were located at the end of the notch. For the unnotched specimen, the two points were apart a distance 


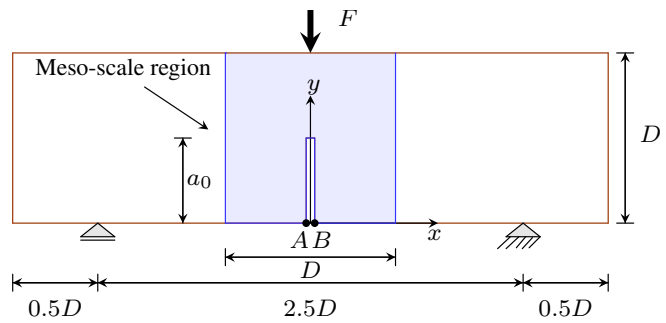

(a) Half-Notched beam

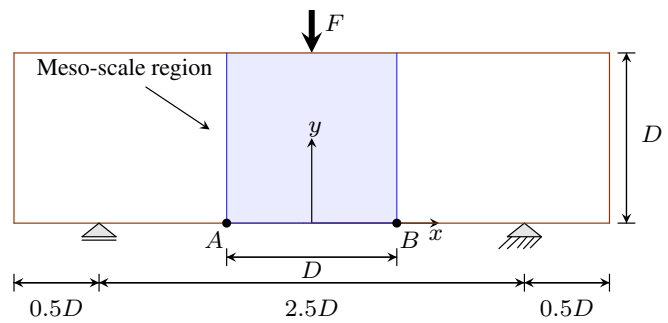

(b) Unnotched beam

Figure 3: Geometries of three-point bending test for (a) notched and (b) unnotched beams. Reproduced from [20].

equal to the beam depth $D$, since the location of the fracture process zone initiating from the surface was indeterminate.

Same as in the experiments, the out-of-plane thickness was kept constant for all sizes and all geometries at $b=50 \mathrm{~mm}$. The notch thickness was fixed equal to zero for consistence with the experimental procedure where the notch was moulded using a thin metal plate of constant thickness. The load and support reactions were applied by means of $5 \mathrm{~mm}$-wide metallic plates.

In order to limit the computation time, the nonlinear mesoscale model is used in the middle part of each beam centered at mid-span where damage is expected, as shown in Figures $3 \mathrm{a}$ and $3 \mathrm{~b}$. The remaining part of the beam is discretized with elastic lattice elements. In this region, the aggregates are not described explicitly. The mechanical response of this part of the lattice corresponds to that of the equivalent homogeneous material. The aggregate volume fraction corresponds to the experimental data , with a cut off for small sizes. Fine aggregates are not explicitly described. They are included in the matrix which is an equivalent homogeneous material made of cement paste and fine aggregates. Disorder due to the heterogeneity of the matrix is considered in the form of a correlated random distribution of mechanical properties. The correlation length is equal to $1 \mathrm{~mm}$. Details may be found in [9].

The model parameters for the three components are summarized in table 1. Such as in Ref. [9], these values were chosen so that the global model results in term of load-CMOD curves for different beam sizes and boundary condi- tions were in agreement with experimental results reported in [14]. However, not all parameters were varied independently of each other to obtain this agreement. Instead, several constraints were applied, motivated by experimental and numerical results reported in the literature. Firstly, the ratio of the stiffnesses for aggregate and matrix was kept constant and equal to two. Furthermore, the tensile strength of matrix was assumed to be twice of the strength of the interfacial transition. These ratios are in the range of the experimental results reported in [16, 25]. Furthermore, the model parameters for the elastic response outside the meso-scale region were chosen so that the response represents the average elastic behaviour of the mesoscale region.

Table 1: Model parameters.

\begin{tabular}{ccccc}
\hline & $E[\mathrm{GPa}]$ & $\nu$ & $f_{t}[\mathrm{MPa}]$ & $G_{f}[\mathrm{~N} / \mathrm{m}]$ \\
\hline \hline Matrix & 44 & 0.33 & 3.8 & 86 \\
\hline Interface & 58.7 & 0.33 & 1.9 & 43 \\
\hline Aggregate & 88 & 0.33 & - & \\
\hline Mean & 63 & 0.33 & - & \\
\hline
\end{tabular}

For each geometry, calculations were repeated with 10 different random fields of aggregates and mechanical properties.

The experimental and numerical results in term of Force vs. CMOD data are presented in [20]. As already discussed in [14], the experimental datapoints are in good agreement with the results obtained via the mesoscopic approach. 


\subsection{Comparison in term of correlation length evolutions}

In this section, we aim at comparing the experimental data and the numerical results in term of evolutions of the correlation length extracted based on the analysis by Ripley's functions and the procedure presented in section 2.3 . To perform the comparison between the experimental and the numerical results, different statements have to be considered:

\section{- Damage event definition.}

The procedure of correlation length extraction presented in section 2.3 is based on the analysis by Ripley's function of a given distribution of damage events. Experimentally, a damage event is associated to a material point producing acoustic emissions upon failure, which have been detected and then localized by at least three acoustic sensors during a load step. Numerically, a damage event is associated to a lattice material point undergoing damage during a load step.

\section{- Loading curve discretization.}

A minimum number of damage events has to be captured to perform the postprocessing. Numerically there is almost no limitation because a lot of damage events are acquired within a loading step. Experimentally, the acoustic emission is much more restrictive because only few acoustic events may be acquired by the technique, especially in the nonlinear prepeak regime. Therefore, the loading curve discretization is determined to ensure to capture enough events experimentally in order to achieve a statistically representative post-processing. Since the first goal of this section is to test the relevance of the meso-model by comparing the numerical results with experimental ones, we adopt the same interval length, which is driven by the experimental minimum. Numerical investigations on correlation length evolutions upon failure based on a finer loading curve discretization are presented alone in part 5 .

\section{- Space discretization.}

Numerically, the space discretization corresponds intrinsically to the lattice discretization. Experimentally, there is an implicit space discretization due to the acoustic sensor resolution and the acoustic emission localization technique resolution. This resolution is of the order of $4 \mathrm{~mm}$ [14]. This means that two acoustic emissions produced at two different material points separated by a distance smaller than this resolution may not be distinguished. This means also that all the acoustic emissions produced in the corresponding vicinity of a material point are seen by the acoustic sensors as a single acoustic emission with an acoustic energy corresponding to the addition of all the individual acoustic energies. Therefore the numerical and experimental data cannot be directly compared since the Ripley's function post-processing is only based on the spatial repartition of a given distribution of point. Experimentally, an acoustic event will count for a single data point in the Ripley's function analysis even if, locally, several material points undergo damage and produce acoustic emissions. This is overcome by tacking into account the intensity of the energy dissipated during each damage event in the post-processing by Ripley's functions. Assuming that the acoustic energy recorded for each event is proportional to the energy dissipated during the corresponding damage event, it is possible to compare experimental and numerical results in term of dissipated energy:

- On one hand, the dissipated energy during damage is obtained numerically from the mesoscale analysis. Maps of dissipated energy have been already computed in [9, 11, 14] and we follow the same procedure. 
The domain to be analyzed is first discretized with a square grid with a cell-size $d=5 \mathrm{~mm}$. Within each cell, the energy dissipation due to damage is computed for each lattice element located in the cell. Then, we sum this energy dissipation for all lattice elements contained in the cell. When a lattice element crosses several cells, the energy is allocated in proportion to the element length within each cell.

- On the other hand, the maps of the distribution of acoustic energy within the same loading increments are computed according to the same discretization. Within an increment, the energy of all the event contained in the same cell is summed up. Due to the localization resolution by acoustic emission technique, less acoustic events than numerical events are detected. Therefore, the size and the discretization of the load increments are determined to ensure to capture enough events experimentally in order to achieve statistically representative post-processing.

Finally, the experimental and numerical energies dissipated within each cell are converted proportionally into a number of points, which are randomly spreaded within the cell. Since the Ripley's function post-processing does not depend on the total number of points (see [20] for details), the post-processing does not depend on this proportionality factor. Practically, the maximum value of the energy (numerical dissipated energy or experimental acoustic energy) is converted into 200 points. After conversion, experimental and numerical evolutions of extracted correlation length may be compared. Note that after conversion, the extracted correlation lengths are smaller than the one extracted without any conversion and they are not directly linked to the fracture process zone size or to the internal length in the sense of nonlocal models because a large number of points are artificially placed close to the macrocrack path corresponding to high energy events. This is acceptable in this section where the first goal is to compare experimental and numerical data. Numerical investigations on correlation length evolutions upon failure without any conversion are presented alone in part 5

Figure 4 presents the comparison between experimental and numerical extracted correlation length with intensity conversion. The correlation length is extracted based on the analysis by Ripley's functions as presented in section 2.3. Even if an important scattering is observed on the experimental data, we observe a global good agreement between the experimental and the numerical results. This means that the numerical model and the Ripley's postprocessing procedure may be used alone to investigate the evolution of the correlation length upon failure.

\section{NUMERICAL INVESTIGATIONS OF COR- RELATION LENGTH EVOLUTIONS UPON FAILURE}

\subsection{Influence of the loading type}

\subsubsection{Response in direct tension}

In this subsection, the correlation length extraction method is applied to a direct tension test. We consider a concrete specimen presenting the same characteristics than the one studied in section 4.1. 

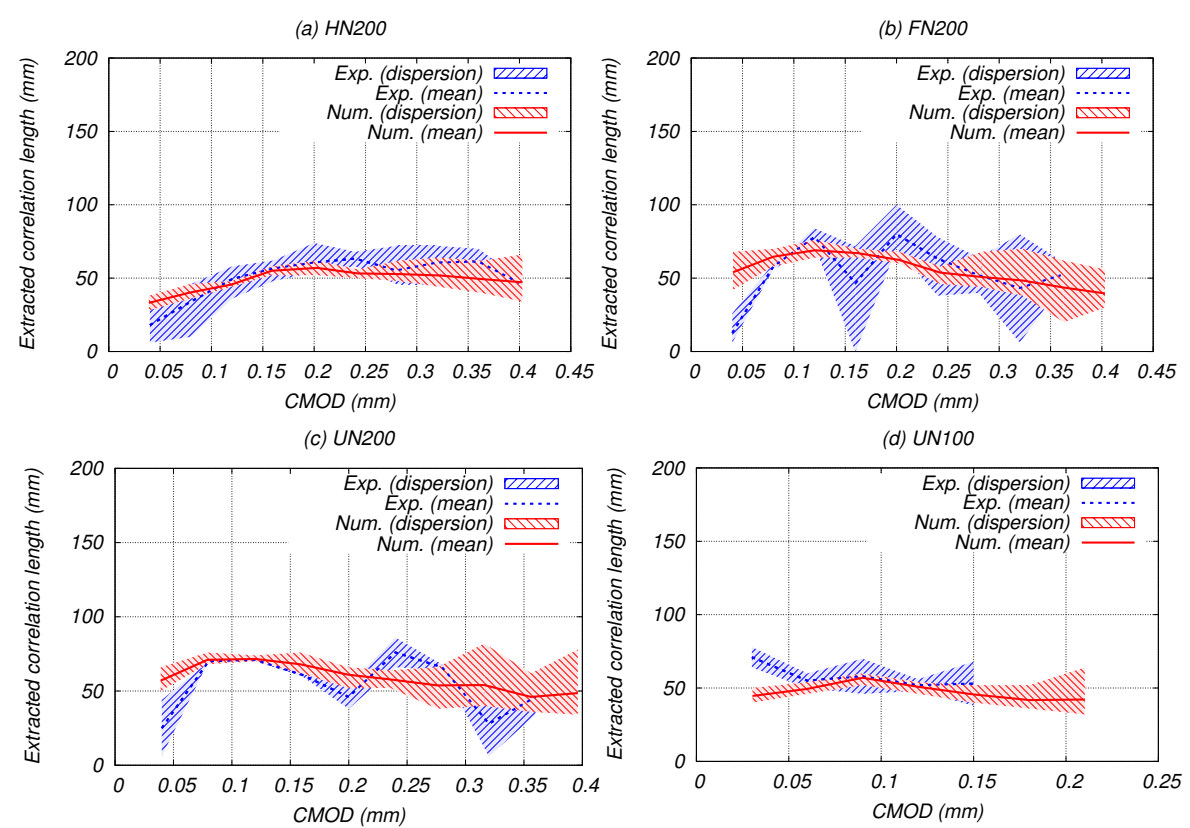

Figure 4: Comparison between experimental and numerical extracted correlation length with intensity conversion. Reproduced from [20].

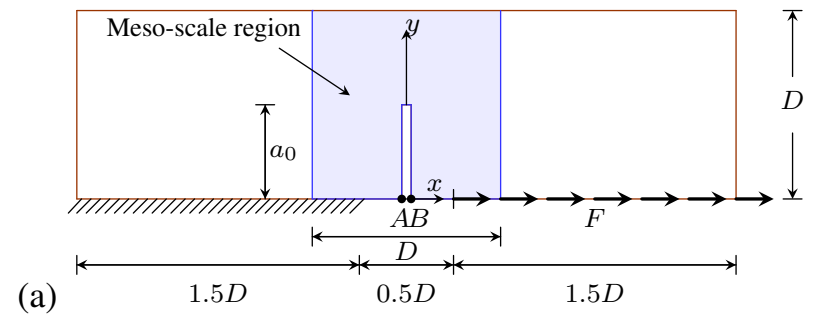

(b)

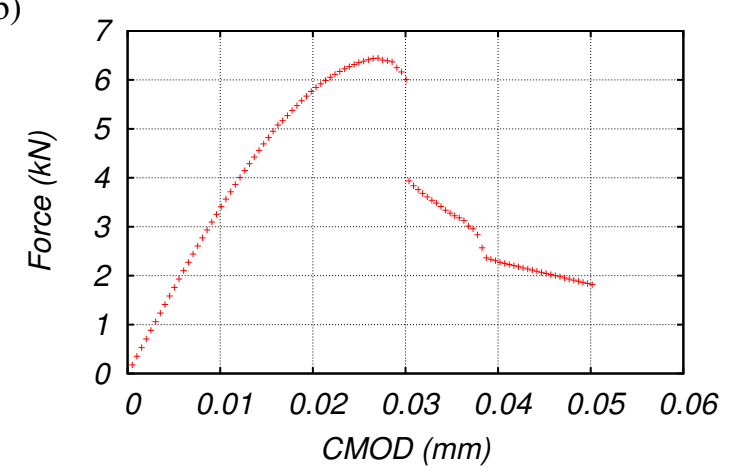

Figure 5: (a) Geometry and (b) Force vs. CMOD curves of the direct tension test $(D=$ $100 \mathrm{~m})$.

Figure 5 presents the tension test geometry and the Force vs. CMOD curves. The specimen is pre-notched from the bottom face to half the depth and the test is CMOD controlled. At each CMOD step, the distribution of incremental damage events is plotted (see figure 6a) and the corresponding Ripley's residual function is

estimated. The correlation length is then extracted based on the analysis by Ripley's functions as presented in section 2.3 .

This correlation length is directly related to the size of the damage zone and therefore to the internal length in a nonlocal continuum setting. The evolution of the extracted correlation length is presented in figure 6. b. In concrete, damage develops at the interface between aggregates and mortar. At the beginning of the test, damage develops and spreads all over the specimen and then the correlation length grows and it would reach eventually the size of the box. However, at $\mathrm{CMOD} \approx 0.01 \mathrm{~mm}$, damage starts to localize within a fracture process zone surrounding the pre-notch tip and the correlation length reaches a plateau. Later on, and as the fracture process zone develops to form a macro-crack, the correlation length decreases. When the macro-crack is fully developed, surrounded by the fracture process zone, the correlation length reaches a new plateau at a value corresponding to four times the larger aggregate size $(\approx 10 \mathrm{~mm})$. 
(a)

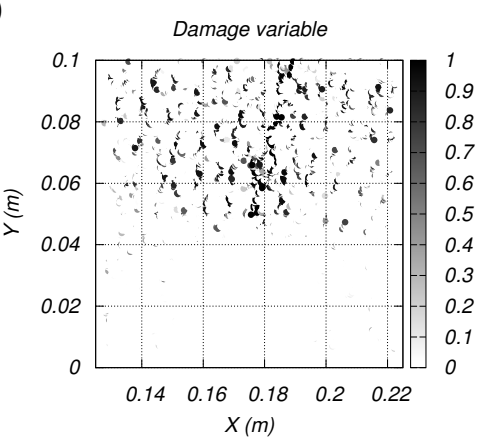

(b)

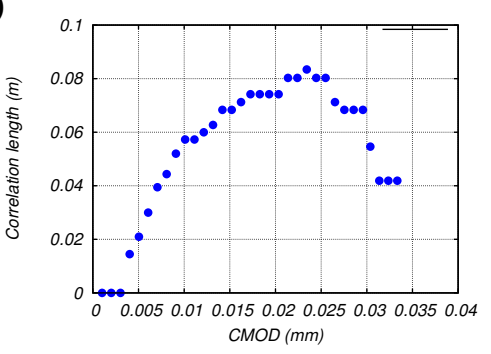

Figure 6: Distribution of damage events for a direct tension test (a) and corresponding correlation length evolution (b).

\subsubsection{Response in bending}

In this subsection, the correlation length extraction method is applied to the three point bending beams presented in section 4.1. We compare here the response of long notch and unnotched specimens with a depth of $100 \mathrm{~mm}$. Geometries are presented in figure 3. The same post processing method is applied: the test are CMOD controlled and at each CMOD step, the distribution of incremental damage events is plotted (see figure $7 \mathrm{a}-\mathrm{b}$ ) and the corresponding Ripley's residual function is estimated. The correlation length is extracted based on the analysis by Ripley's functions as presented in section 2.3. The evolution of the extracted correlation length is presented in figure 7.c. (a)

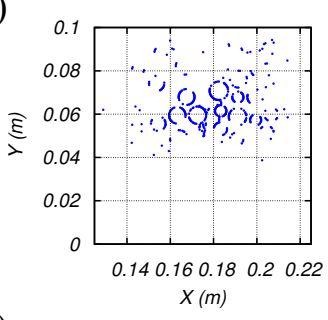

(b)

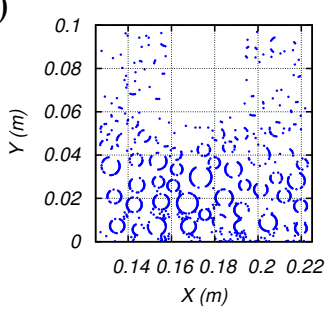

(c)

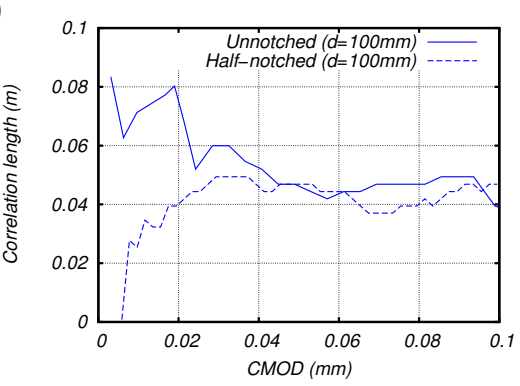

Figure 7: Responses in bending: (a) Damage distribution at peak for the long notch specimen; (b) Damage distribution at peak for the unnotched specimen; (c) Evolution of the extracted correlation lengths for both specimen.

For the notched specimen, the evolution of damage is similar to what has been observed in direct tension. The pre-notch trigs the damage localization and the correlation length grows to reach a plateau at a value corresponding to four/five times the larger aggregate size $(\approx$ $10 \mathrm{~mm}$ ). However, during the initiation of failure, damage does not spread over the whole specimen because of the bending strain gradient. That is the reason why only a growing phase is observed before the plateau. For the unnotched specimen, the damage evolution is totally different. Since there is no pre-notch, the damage localization is not trigged and damage spreads on the bottom surface of the specimen. Therefore, the correlation length is equal to the analysis box size at the beginning at damage initiation. At some point, a macro-crack will emerges from the bottom face and propagates surrounded by a fracture process zone. 
The correlation length is decreasing from the analysis box size to reach the same plateau observed for a notched specimen at a value corresponding to four/five times the larger aggregate size $(\approx 10 \mathrm{~mm})$.

\section{CONCLUding REMARKS}

We have presented a detailed analysis based on Ripley's functions of the cracking process at the mesoscale of concrete specimen, both numerically and experimentally. The computational model is a lattice-based approach which already proved to be able to capture size effect test data for notched and unnotched bending beams and the force v.s. CMOD response as well (see [9]). Moreover, comparison with experiments coupled with acoustic emission analyses proved also that the mesoscale model is representative of the local process of quasibrittle failure in term of dissipative energy maps and histograms of relative distances between damage events (see [14]).

The following concluding statements can be made:

- The post processing with Ripley's function provides indicators of the randomness of a distribution of events.

- It has been shown that a correlation length, which may possibly be linked to an internal length in the sense of non local models, may be extracted from the Ripley's function analyse applied to damage mechanics. However, the exact correspondance between the extracted correlation length and a nonlocal model internal length remains to be derived.

- The evolutions of this extracted correlation length upon failure have been presented. Comparisons between numerical data based on mesoscale lattice modeling and experimental data where damage events were localized by acoustic emission techniques were performed. Even if an important scattering is observed on the experimental data, we observed a global good agreement between the experimental and the numerical results. This means that the numerical model and the Ripley's post-processing procedure may be used alone to investigate the evolution of the correlation length upon failure.

- Numerical investigations have been performed on both direct tension and three point bending specimens. The results show that the extracted correlation length is not constant during failure and significant differences may be observed depending on the type of loading applied to the same specimen.

This conclusion opens the path for further analyses of the fracture process, solely based on numerical analyses with the mesoscale model. From theses studies, a better understanding of the correlations between damage events, that should result into non local continuum modeling at the macroscale, is expected.

\section{ACKNOWLedgments}

The authors wish to acknowledge the University of Bordeaux for the use of the cluster AVAKAS.

\section{REFERENCES}

[1] SY Alam, Saliba J, and Loukili A. Fracture examination in concrete through combined digital image correlation and acoustic emission techniques. Construction and Building Materials, 69:232-242, 2014.

[2] P. J. Diggle and A. G. Chetwynd. SecondOrder Analysis of Spatial Clustering for Inhomogeneous Populations. Biometrics, 47(3):1155-1163, 1991.

[3] Philip M Dixon. Ripleys $\mathrm{K}$ function. In Abdel H El-shaarawi and Walter W Piegorsch, editors, Encyclopedia of Environmetrics, volume 3 , volume 3 , pages 1796-1803. John Wiley \& Sons, Ltd., Chichester, 2002. 
[4] Kai Duan, Xiaozhi Hu, and F.H. Wittmann. Boundary effect on concrete fracture and non-constant fracture energy distribution. Engineering Fracture Mechanics, 70(16):2257 - 2268, 2003.

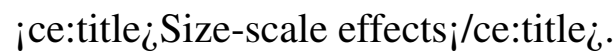

[5] Richard P Duncan. Flood Disturbance and the Coexistence of Species in a Lowland Podocarp Forest, South Westland, New Zealand Author(s):. Journal of Ecology, 81(3):403-416, 1993.

[6] Cédric Giry, Frédéric Dufour, and Jacky Mazars. Stress-based nonlocal damage model. International Journal of Solids and Structures, 48(25-26):34313443, 2011.

[7] François Goreaud and Raphaël Pélissier. On explicit formulas of edge effect correction for ripley's k-function. Journal of Vegetation Science, 10(3):433-438, 1999.

[8] S. Granger, A. Loukili, G. PijaudierCabot, and G. Chanvillard. Experimental characterization of the self-healing of cracks in an ultra high performance cementitious material: Mechanical tests and acoustic emission analysis. Cement and Concrete Research, 37(4):519 - 527, 2007.

[9] P. Grassl, D. Grégoire, L. B. RojasSolano, and G. Pijaudier-Cabot. Mesoscale modelling of the size effect on the fracture process zone of concrete. International Journal of Solids and Structures, 49(13): 1818-1827, 2012.

[10] Peter Grassl. A lattice approach to model flow in cracked concrete. Cement and Concrete Composites, 31(7):454 - 460, 2009.

[11] Peter Grassl and Milan Jirásek. Mesoscale approach to modelling the fracture process zone of concrete subjected to uniaxial tension. International Journal of
Solids and Structures, 47(7-8):957-968, 2010.

[12] D. Grégoire, L. B. Rojas-Solano, and G. Pijaudier-Cabot. Continuum to discontinuum transition during failure in nonlocal damage models. International Journal for Multiscale Computational Engineering, 10(6):136, 2012.

[13] D. Grégoire, L. B. Rojas-Solano, and G. Pijaudier-Cabot. Failure and size effect for notched and unnotched concrete beams. Int. J. Numerical and Analytical Methods in Geomechanics, 37(10):14341452, 2013.

[14] Grégoire, D. and Verdon, L. B. and Lefort, V. and Grassl, P. and Saliba, J. and Regoin, J.-P. and Loukili, A. and PijaudierCabot, G. Mesoscale analysis of failure in quasi-brittle materials: comparison between lattice model and acoustic emission data. International Journal of Numerical and Analytical Methods in Geomechanics, 39(15):1639-1664, 2015.

[15] K Haidar, G Pijaudier-Cabot, J Dubé, and A Loukili. Correlation between the internal length, the fracture process zone and size effect in model materials. Materials and Structures, 38(2):201-210, 2005.

[16] Thomas T. C. HSU and Floyd O. Slate. Tensile Bond Strength Between Aggregate and Cement Paste or Mortar. ACI Journal Proceedings, 60(4):465-486, 1963.

[17] A. Krayani, G. Pijaudier-Cabot, and F. Dufour. Boundary effect on weight function in nonlocal damage model. Engineering Fracture Mechanics, 76(14):2217-2231, 2009.

[18] Christian La Borderie, Claire Lawrence, and Abdellah Menou. Approche mésoscopique du comportement $\mathrm{du}$ béton: Apport de la représentation 
géométrique. Revue européenne de génie civil, 11(4):407-421, 2007.

[19] Eric N. Landis. Micro-macro fracture relationships and acoustic emissions in concrete. Construction and Building Materials, 13(12):65 - 72, 1999.

[20] Vincent Lefort, Gilles Pijaudier-Cabot, and David Grgoire. Analysis by ripleys function of the correlations involved during failure in quasi-brittle materials: Experimental and numerical investigations at the mesoscale. Engineering Fracture Mechanics, 147:449 - 467, 2015.

[21] Koji Otsuka and Hidehumi Date. Fracture process zone in concrete tension specimen. Engineering Fracture Mechanics, 65(23): $111-131,2000$.
[22] B . D . Ripley. Modelling Spatial Patterns. Journal of the Royal Statistical Society. Series B (Methodological), 39(2):172-212, 1977.

[23] Nancy E Stamp and Jeffrey R Lucas. Spatial Patterns and Dispersal Distances of Explosively Dispersing Plants in Florida Sandhill Vegetation. Journal of Ecology, 78(3):589-600, 1990.

[24] Antoinette Tordesillas, Sebastian $\mathrm{Pu}$ cilowski, Luc Sibille, François Nicot, and Félix Darve. Multiscale characterisation of diffuse granular failure. Philosophical Magazine, 92(36):4547-4587, 2012.

[25] JGM van Mier. Fracture processes of concrete. Assessment of material for fracture models. CRC Press, Boca Raton, USA, 1997. 